\title{
Lagerbildung und Verständigung in der kritischen Normenforschung. \\ Eine Einleitung
}

Katharina Glaab, Antonia Graf und Stephan Engelkamp

\section{Von Lagerfeuern und Rauchzeichen}

Betrachtet man die Entwicklung der Internationalen Beziehungen (IB) anhand ihrer großen Debatten, kann man den Eindruck einer in verschiedene (wissenschaftliche) Lager gespaltenen Disziplin gewinnen (Lapid 1989; Wæver 2010). So macht Christine Sylvester einen ausgeprägten Hang zur Lagerbildung in den IB aus: demnach differenzieren sich unterschiedliche Ansätze der IB nicht nur immer weiter intern aus, sie grenzen sich dabei auch deutlich - wie an Lagerfeuern einzelner Lager - voneinander ab. An diesen Lagerfeuern erzählen sich WissenschaftlerInnen Geschichten darüber, wie die eigene Gruppe entstanden ist und inwiefern sie sich von den anderen unterscheidet (Sylvester 2007, 2013). Diese Erzählungen wirken überaus identitätsstiftend. Sie ermöglichen den Mitgliedern eines Lagers die Identifizierung mit und Abgrenzung zu einer Gruppe akademischer (Gegen-)SpielerInnen. Zuweilen erscheinen die zugeschriebenen Unterschiede zwischen diesen Gruppen wie tiefe Gräben, aber gelegentlich kommt es zwischen den Lagern auch zu Austauschprozessen und zur Brückenbildung (siehe etwa die Versuche, einen middle ground zwischen rationalistischen und konstruktivistischen Ansätzen zu finden, vgl. Adler 1997; Checkel 1997; Risse 2003; Zürn/Checkel 2005). Die Lagerbildung kann demnach auch als Zwischenstufe zur Weiterentwicklung einer Disziplin gelesen werden, die vor allem dann zu einem Erkenntnisfortschritt beiträgt, wenn die »Rauchzeichen« des jeweils anderen Lagerfeuers gedeutet und verstanden werden können. ${ }^{1}$

Als Beispiel für Deutungsschwierigkeiten mag der Austausch zu kritischer Normenforschung im Forum der Zeitschrift für Internationale Beziehungen dienen (Engelkamp et al. 2012; Ulbert 2012; Deitelhoff/Zimmer-

1 Den Hinweis auf Christine Sylvesters Lager-Metapher und ihr Bild vom Dialog zwischen IB-Ansätzen als Deuten von Rauchzeichen verdanken wir Sassan Gholiagha. 
mann 2013; Engelkamp et al. 2013; Hofius et al. 2014). Hier scheint es nicht nur um die Bewertung der Stärken und Schwächen konstruktivistischer Normenforschung an sich zu gehen, sondern auch um die Frage, was kritische politikwissenschaftliche Forschung ausmacht. Selbst innerhalb des breiten Feldes der konstruktivistischen Forschungen, also all denjenigen Ansätzen, die globale Politik als durch Normen, Ideen oder Kultur sozial konstruiert verstehen, zeigen sich Tendenzen zu einer zunehmenden Lagerbildung, die neben den Verständigungsschwierigkeiten aber auch die Möglichkeit zum Erkenntnisgewinn birgt, wenn die Rauchzeichen gelesen werden.

Dementsprechend sind konstruktivistische Ansätze zur Normenforschung, so unsere Annahme, immer auch ein Ort des Austausches zwischen verschiedenen methodologischen Forschungsparadigmen gewesen. Gerade konstruktivistische NormenforscherInnen haben versucht, Rauchzeichen auszusenden, um mit ihren KollegInnen aus dem rationalistischen Forschungsprogramm im Gespräch zu bleiben (vgl. etwa Klotz 1995; Finnemore/Sikkink 1998). Der Aufgabe des Lesens von Rauchzeichen als Voraussetzung für die Suche nach Verständigungsmöglichkeiten, aber auch für die Identifizierung von Unterschieden ist der vorliegende Band gewidmet. NormenforscherInnen aus unterschiedlichen Lagern betreiben zum einen Forschung über die empirische Wirkungsweise von Normen, aber auch metatheoretische Forschung über Normenforschung selbst. Für beide Perspektiven und die voranschreitende Verständigung darüber gibt es einen großen Bedarf, da sich gerade die Normenforschung in den letzten 30 Jahren als eine wichtige Perspektive konstruktivistischer Forschung entwickelt hat. Allerdings kann angesichts der Bandbreite unterschiedlicher Ansätze, die im weiteren Sinne zu Normen in der internationalen Politik forschen, kaum von , der ${ }^{6}$ Normenforschung im Singular gesprochen werden. Stattdessen scheinen sich gerade bei diesem Forschungsfeld unterschiedliche Konstruktivismen und verschiedene Verständnisse davon, was (kritische) Forschung über Normen ausmacht, zu versammeln (vgl. hierzu den Befund von Bastian Loges in diesem Band).

Diese Heterogenität von Normenforschungen im Plural wurde uns an dem Rücklauf auf unseren Call for Paper zum 4. Workshop des Netzwerks Kritische Normenforschung ${ }^{2}$ anhand der Vielfalt theoretischer, methodischer und thematischer Zugänge besonders deutlich. Die Bandbreite reichte da-

2 Das Netzwerk Kritische Normenforschung ist aus der IB-Nachwuchstagung im Mai 2010 hervorgegangen. Die Workshops in Frankfurt (2010), Duisburg (2011) und Hamburg (2011) gingen dem vierten Treffen in Rothenberge bei Münster vor- 
bei von neo-institutionalistischen Ansätzen über wissens- und raumsoziologische Untersuchungen bis zu von Foucault oder Laclau und Mouffe inspirierten poststrukturalistischen Vorschlägen. Zudem wiesen die Beiträge die von Sylvester konstatierten Merkmale der Lagerbildung auf: den Bezug auf jeweils eigene Gründungstexte und AutorInnen, unterschiedliche Interpretationen derselben und die Pflege der eigenen Konzepte und Begrifflichkeiten. Die Lagerbildung führte zu spezifischen Nuancierungen etablierter Lesarten bis hin zu teilweise diametral entgegengesetzten Interpretationen derselben Texte und Wissensbestände. NormenforscherInnen scheinen sich - zumindest im deutschen Kontext - gleich an mehreren dieser akademischen Lagerfeuer eingerichtet zu haben. Zugleich wurden auf dem Workshop in Rothenberge in unterschiedlichen Lagern oftmals ähnliche Probleme verhandelt, wobei die konkreten Antworten - trotz terminologischer Unterschiede - häufig nicht so sehr voneinander abwichen, wenn es um die konkrete empirische Umsetzung ging (vgl. hierzu auch Risse 2003).

Wie im Folgenden gezeigt wird, wendet sich die Kritik, die in der Zeitschrift für Internationale Beziehungen an IB-Normenforschung formuliert wurde, oftmals gegen funktionalistische und eurozentrische Vorstellungen von normativem Wandel, die Vernachlässigung der Entstehungsbedingungen von normativen Ordnungen, die Ausblendung von Historizität und Macht sowie eine mangelnde Reflexion der Normativität der Normenforschung als politischer Praxis. Kritische Interventionen in Auseinandersetzung mit frühen Arbeiten konstruktivistischer NormenforscherInnen wurden zuvor vor allem im anglo-amerikanischen Raum geäußert und waren ebenfalls noch relativ stark in der Lagerfeuer-Logik verhaftet (vgl. auch Berenskötter 2014: 134; Stritzel 2014: 143 sowie Wiener 2014: 151).

Diese Kritik verweist auf eine ganze Reihe von möglichen Entwicklungspfaden, die NormenforscherInnen einschlagen können, wenn ihre Forschung durch metatheoretische Reflexion einen konstruktiven Dialog ermöglicht (Kratochwil 2007). Bislang werden diese kritischen Diskussionen in der deutschen IB jedoch kaum rezipiert. Ausnahmen davon bilden hegemoniekritische Ansätze von feministischer Seite (vgl. Brabandt et al. 2002) und eben die Diskussion unterschiedlicher Forschungsperspektiven zu kritischer Normenforschung in der ZIB. Gerade in der ZIB-Diskussion

aus, aus dem der vorliegende Band entstanden ist. Die gemeinsame Arbeit und Diskussionen aller Treffen waren grundlegend für diesen Band. Unser Dank gilt allen Beteiligten, insbesondere Doris Fuchs, und der Fritz Thyssen Stiftung für die finanzielle Unterstützung des Workshops und dieses Bandes. 
und den Repliken von Cornelia Ulbert, Nicole Deitelhoff und Lisbeth Zimmermann sowie Maren Hofius, Jan Wilkens, Hannes Hansen-Magnusson und Sassan Gholiagha zeigt sich jedoch, dass große Uneinigkeit darüber besteht, was kritische und insbesondere reflexive Forschung ausmacht (Deitelhoff/Zimmermann 2013; vgl. auch Koddenbrock 2015 sowie Anderl/Wallmeier 2018). An dieser Stelle möchten wir als HerausgeberInnen deutlich machen, dass wir in diesem Band nicht festlegen, was als kritisch anzusehen ist. Dies kann und soll nicht Anspruch eines Sammelbands über Normenforschungen im Plural sein. Wie die LeserInnen in den folgenden Kapiteln sehen werden, betreten die AutorInnen dieses Bandes durchaus unterschiedliche Pfade und diese Pluralität wird von uns begrüßt.

Vor diesem Hintergrund stellten sich uns als HerausgeberInnen des vorliegenden Sammelbands zwei zentrale Fragen: Wie verhält sich Forschung über Normen zu reflexiven und eher metatheoretischen Arbeiten über Normenforschung? In welcher Weise lässt sich auf dieser metatheoretischen Basis eine Verständigung über unterschiedliche kritische Ansätze der Normenforschung bezeichnen, die neue Wege abbildet?

\section{Frühe konstruktivistische Normenforschung als Ort des Austausches}

Die frühe konstruktivistische Normenforschung erschien in den späten 1980er und 1990er Jahren als eine reizvolle Alternative im Hinblick auf die Auslassungen eines im weitesten Sinne als rationalistisch geprägten IB-Forschungsprogramms (Keohane 1988). Indem KonstruktivistInnen Normen als bislang vernachlässigten Einflussfaktor zur Erklärung von Akteurshandeln in klassische Forschungsprogramme einbezogen, bereiteten sie der Weiterentwicklung einer ganzen Reihe von Forschungsansätzen den Weg. Die Normenforschung konnte sich so als fester Bestandteil des Mainstreams in den Internationalen Beziehungen etablieren (Guzzini 2000). Sie wird heute weitgehend als Teil einer konstruktivistischen Perspektive wahrgenommen, welche sich Ende der 1980er Jahre als Kritik an rationalistischen Ansätzen in den IB entwickelte (vgl. etwa Adler 1997; Checkel 1998; Fearon/Wendt 2008; Widmaier/Park 2012 geben einen Überblick über neuere konstruktivistische Beiträge; vgl. aber auch die eher rationalistisch angelegten Studien von Schimmelfennig 2001, 2005). Nach dem Ende des Ost-West-Konflikts und dem Zusammenbruch der Sowjetunion zeigte sich, dass die bestehenden rationalistischen Erklärungsangebote von globalem Wandel anhand von strukturellen und materiellen Bedingungen an ihre konzeptionellen Grenzen gelangt waren. Konstruktivistische For- 
schung betonte demgegenüber die kausale Bedeutung ideeller Faktoren (Goldstein/Keohane 1993), etwa bei der Definition politikrelevanter Probleme oder bei der Wahl von Lösungsstrategien, wie Ulbert (1997) in ihrer Studie zur Einrichtung der internationalen Klimarahmenkonvention zeigte. Das Handeln von AkteurInnen in der globalen Politik wurde somit als durch soziale Konstruktionen wie Identität, Kultur oder Ideen beeinflusst verstanden (Klotz 1995; Katzenstein 1996b).

Insbesondere die konstruktivistische Forschung in den IB entwarf mit dem Fokus auf Normen, verstanden als "standard of appropriate behaviour for actors with a given identity" (Finnemore/Sikkink 1998: 891, vgl. auch Katzenstein 1996a: 5), ein differenzierteres Bild von politischem Wandel als dies rationalistische Ansätze bis dato leisteten. Normen spielen dabei eine maßgebliche Rolle, indem sie die Identität und die damit zusammenhängenden Interessen eines Akteurs konstituieren und gleichzeitig einschränken (Kratochwil 1991; Wendt 1999; Krasner 1999). Während jedoch die regulierende Wirkung von Normen insbesondere in die rationalistische Institutionentheorie Einzug gehalten hat, beschäftigt sich die konstruktivistisch orientierte Normenforschung vornehmlich mit der konstituierenden Wirkung von Normen auf die Interessen und Identitäten von AkteurInnen, die einer Logik der Angemessenheit folgt (March/Olson 1998, Risse 2003). Hier wird insbesondere die Rolle von Normen für die Herausbildung globaler Ordnungsstrukturen, etwa im Bereich der Menschenrechte, der Sicherheits- oder der Umweltpolitik untersucht (Zürn et al. 2007, Wallbott/Schapper 2017).

Frühe konstruktivistische Erklärungen von Entstehung und Wandel internationaler normativer Ordnungen setzen dabei nicht alleine auf struktureller Ebene an, sondern betrachten auch die Rolle von lokalen und transnationalen AkteurInnen (Keck/Sikkink 1998; Checkel 1999; Acharya 2004; Risse 2006). Die Entstehung von Normen wird somit durch das aktive Handeln von AkteurInnen ermöglicht, den sogenannten NormunternehmerInnen (Finnemore/Sikkink 1998). Transnational organisierte Nichtregierungsorganisationen bringen in dieser Lesart politischen Wandel hervor, indem sie Öffentlichkeit her- und Informationen bereitstellen, erfolgreich ihre Themen auf die internationale Agenda setzen, aber auch durch Ausübung von politischem Druck in Form von Naming-and-Shaming-Strategien (Deitelhoff 2006). In diesen Ansätzen wird die Institutionalisierung von Normen auf internationaler und nationaler Ebene als Lern- und Überzeugungsprozesse konzeptualisiert, wobei diese Mechanismen zumeist zu einer Internalisierung globaler Normen in verschiedenen lokalen Kontexten führen (Finnemore/Sikkink 1998; Risse et al. 1998). 
Konstruktivistische Normenforscher wie Jeffrey Checkel oder Amitav Acharya, die stärker die Rolle von AkteurInnen in den Blick nehmen, weisen in diesem Zusammenhang auf die Frage nach der konsensualen Anbindung einer internationalen Norm an lokale Werte und Normen hin (Checkel 1997; Acharya 2004; 2011a). Risse und Sikkink (1999) begegnen diesem Hinweis mit einem Fokus auf kommunikative Praktiken der Überzeugung, die eine Norm diskursiv legitimieren. Dieser Ansatz hat insbesondere in der deutschsprachigen konstruktivistischen Normenliteratur weiten Anklang gefunden, so etwa in viel rezipierten Studien zur Verbreitung von Menschenrechten (Risse et al. 1999) oder zum Internationalen Strafgerichtshof (Deitelhoff 2006). So entstand ein fruchtbares Forschungsprogramm, in dem ideelle Faktoren wie Ideen, Identität und Kultur eine wichtige Rolle für die Erklärung internationaler Politik spielten. Dabei blieb gerade konstruktivistische Normenforschung immer auch anschlussfähig an den rationalistischen Mainstream der Internationalen Beziehungen. Diese »Brückenstellung« zwischen rationalistischen und konstruktivistischen Ansätzen hat zu neuen und innovativen theoretischen Erkenntnissen in den Internationalen Beziehungen geführt (Risse 2003).

\section{Kritische Ansätze konstruktivistischer Normenforschung}

Das integrative Potenzial von Normen in Auseinandersetzung mit der rationalistischen IB-Forschung lässt Konfliktlinien zwischen KonstruktivistInnen in der Normenforschung besonders deutlich werden (Wiener 2003). Mit der zunehmenden Öffnung der IB für postpositivistische Ansätze und dem damit einhergehenden Import von Theorien und interpretativen Verfahren wurden den frühen konstruktivistischen Arbeiten über Normen immer wieder Auslassungen und Inkonsistenzen vorgehalten, die dazu geführt haben, dass sich eine Reihe von unterschiedlichen konstruktivistischen Normenforschungen etabliert haben. ${ }^{3}$ Die Weiterentwicklung des konstruktivistischen Normenforschungsprogramms durch ForscherIn-

$3 \mathrm{Zu}$ einer Auseinandersetzung siehe hierzu aktuell die bereits erwähnten ZIB-Beiträge zu kritischer Normenforschung für den deutschsprachigen Bereich (Engelkamp et al. 2012; Ulbert 2012; Deitelhoff/Zimmermann 2013; Engelkamp et al. 2013; Hofius et al. 2014) sowie die Foren in International Studies Perspectives (Epstein 2012a; Widmaier/Park 2012; Epstein 2012b; MacKenzie/Sesay 2012; Inayatullah/Blaney 2012) und International Theory (Epstein et al. 2014; Epstein 2014; Zarakol 2014; Gallagher 2014; Shilliam 2014; Jabri 2014) sowie von Hofferberth/Weber 2015. 
nen in Auseinandersetzung mit frühen Arbeiten, die sich in unserer Lesart eher dem konstruktivistischen Mainstream der IB zuordnen lassen, drückt sich - wenig verwunderlich - zunächst einmal in Form einer breiten Bandbreite ontologisch, epistemologisch und methodologisch informierter Kritik aus. Die Auslassungen, die von kritischen Ansätzen in diesem Zusammenhang problematisiert werden, beziehen sich auf den Status des Lokalen in internationalen Normen, einen potenziellen Eurozentrismus, die Vernachlässigung von Sprache und den darin materialisierten Machtbeziehungen, die Unterschätzung andauernder Umstrittenheit von Normen und das Fehlen einer reflexiven Perspektive im Hinblick auf die Diskurspositionen der Forschenden und der eigenen Normativität.

\section{Normen zwischen internationaler und lokaler Adaption}

Ein erster Kritikpunkt kritischer konstruktivistischer Ansätze in den IB an der frühen konstruktivistischen Normenliteratur bemängelt, dass der analytische Fokus auf der bloßen Verbreitung internationaler Normen liege (Wiener 2004, 2009). Normativer Wandel wird dabei - so die Kritik - zumindest implizit als dichotome Normenübernahme oder -ablehnung konzeptualisiert (Zimmermann 2016). Kritische KonstruktivistInnen argumentieren weiter, dass diese Forschungsperspektive lokale Kontexte in Adaptionsprozessen vernachlässige und insbesondere Fragen von Macht kaum behandelt werden. Sozialisierungs- (Risse et al. 1999), Diffusions(Holzinger et al. 2007) oder Überzeugungsprozesse (Deitelhoff 2006) erfassen somit nur unzureichend den Austausch, der zwischen internationalen Normen und lokalen Vorstellungen und Praktiken bestehe (Fuchs/Glaab 2011; MacKenzie/Sesay 2012).

Dieser Kritikpunkt ist häufig verbunden mit dem Vorwurf, dass weite Teile der IB-Normenforschung oftmals funktionalistische und eurozentrische Vorstellungen von normativem Wandel aufweisen und dabei die Entstehungsbedingungen von normativen Ordnungen vernachlässigen (vgl. etwa Engelkamp et al. 2012; Epstein 2012b, 2014). Jüngere Arbeiten der konstruktivistischen Normenforschung haben diese Kritikpunkte aufgegriffen und argumentieren, dass Normen nicht einfach diffundieren, sondern in nationale und lokale Kontexte übersetzt werden (Zwingel 2011; 2016; Wiener/Liste 2014). Hierbei verweist zum Beispiel Susanne Zwingel auf die Arbeiten von Acharya, der zeigt, dass Normen immer auch in Lokalisierungsprozesse eingebunden sind (Acharya 2004, 2011b). Ausgehend von dieser Kritik haben sich AutorInnen mit der Aushandlung der Bedeutungen von Normen nach ihrer formalen legalen Übernahme beschäftigt 
(van Kersbergen/Verbeek 2007; Zimmermann 2017). Die postkoloniale Kritik wird insbesondere von Lokalisierungsansätzen aufgegriffen, die etwa Erkenntnisse aus der Sozialanthropologie einbeziehen und explizit nach lokaler agency, epistemologischer Gewalt und den komplexen Austauschbeziehungen fragen, die mit eurozentrischen Konzeptualisierungen von normativem Wandel aus dem Blick geraten (Platenkamp 2007; 2013; Zimmermann 2016).

\section{Umstrittenheit von Normen}

Ein zweiter Kritikpunkt greift die den Modellen inhärente Konzeptualisierung von normativem Wandel auf und kritisiert, dass die erste Generation der konstruktivistischen Normenforschung von einem weitgehend stabilen Konzept von Normen ausgeht, die von einem Staat zum nächsten diffundieren. Normen werden in dieser Perspektive, so Krook und True (2012), als "Dinge« betrachtet. Dagegen betonen eher prozess-orientierte ForscherInnen wie Antje Wiener, dass Normen immer umstritten und veränderlich sind (Wiener 2009, 2010; vgl. auch Sandholtz 2008, van Kersbergen/Verbeek 2007 und Niemann/Schillinger 2017). In dieser Sicht stellt sich der Aushandlungsprozess zwischen globalen und lokalen Normen als ein diskursiver Re-Konstituierungsprozess dar, in dem die Konstruktion von Bedeutungen durch soziale Praktiken, also »meaning-in-use (Milliken 1999; Wiener 2009; Hansen-Magnusson et al. 2020; Hansen-Magnusson 2020) betrachtet werden müsse. ${ }^{4}$

\section{Sprache und Macht als konstituierendes Merkmal}

Ein dritter Kritikpunkt an konstruktivistischen Beiträgen zielt auf die methodologischen Grundlagen empirischer Normenforschung. So betonen ForscherInnen jenseits der sogenannten Via Media des moderaten Konstruktivismus die zentrale Bedeutung von Sprache (Engelkamp/Glaab 2015). Maja Zehfuss (1998; 2002) argumentiert, dass ihr wesentlicher Unterschied in der Zuordnung und Würdigung des theoretischen Rangs von Sprache besteht. So differenziert sie in Bezug auf Wendt, Onuf und Kratochwil zwischen denjenigen konstruktivistischen Ansätzen, in denen Wirk-

4 Vgl. hierzu auch Holzscheiter (2018) sowie das Forum zu Normrobustheit und Umstrittenheit im Journal of Global Security Studies, Band 4, Heft 1, Januar 2019. 
lichkeit durch symbolische Handlungen (Wendt) geschaffen wird, und denen, die die Produktion von Bedeutung durch Sprechakte (Onuf und Kratochwil) untersuchen (Zehfuss 1998, 2002). Mit dem Fokus auf Sprechakte gehen eher poststrukturalistisch orientierte AutorInnen davon aus, dass alles Soziale diskursiv vermittelt ist. Entsprechend liegt hier der Analysefokus auf der Untersuchung von performativen und symbolischen Handlungen als diskursive Praktiken. Dabei stellt sich die Frage, wie ein bestimmtes Wissen über die Welt als wahr anerkannt wird. So thematisieren AutorInnen wie Der Derian und Shapiro (1989), Campbell (1992), Walker (1993), Doty (1996) oder Zehfuss (2002) in ihren Arbeiten die zentrale Bedeutung von Macht für die soziale Konstruktion von Wirklichkeit. Sie fragen explizit nach den Interaktionen zwischen Erkenntnistheorie und Handlungserklärung und nehmen somit den Wissens-Macht-Nexus in den Blick. Aufgabe der kritischen Analyse ist es, die Entstehung von Wissensbeständen innerhalb eines Diskurses aufzudecken, mit denen eine bestimmte Lesart der Realität denkbar und sagbar wird, während andere Interpretationen marginalisiert werden. Für die Normenforschung bedeutet dies zu hinterfragen, wessen Normen als gültig anerkannt werden und warum (Epstein 2008, 2011). Poststrukturalistische Ansätze setzen hier an und zeigen sowohl die Kontingenz normativer Gefüge als auch alternative Handlungsmöglichkeiten auf (Renner 2013).

\section{Reflexivität in der Normenforschung}

Ein letzter Kritikpunkt, der insbesondere von feministischen IB-ForscherInnen (Sylvester 2000; Brabandt et al. 2002; Ackerly/True 2008a, 2008b) und kritischen ForscherInnen aus dem Bereich der postkolonialen Studien (vgl. Inayatullah/Blaney 2004; Agathangelou/Ling 2009; Grovogui 2010) aufgebracht wurde, fragt nach der kritischen Rolle der Wissenschaft selbst. Hier geraten Forschungsfragen nach Artikulation und Widerstand in den Blick, die direkt auf die normative Selbstposition der Forschenden zielen (vgl. etwa Diez 2005 zur »normative power Europe«) und die Ausblendung von Historizität und Macht sowie eine mangelnde Reflexion der Normativität der Normenforschung selbst thematisieren (Epstein 2014). Zudem hinterfragen eher poststrukturalistisch inspirierte kritische Ansätze die Dispositionen, die der IB-Normenforschung zugrunde liegen (Neumann/ Sending 2010; Epstein 2012a; Engelkamp et al. 2012, Graf 2016). Sie weisen darauf hin, dass eine Tendenz zur Beschäftigung mit liberalen Normen wie Menschen- oder Frauenrechten herrsche, während deren historische Möglichkeitsbedingungen wie Sklaverei oder Kolonialismus kaum be- 
trachtet werden (Rosert/Schirmbeck 2007; Panke/Petersohn 2012; Shilliam 2014; Vitalis 2015, Henderson 2015). Eine kritische Reflexion der eigenen wissenschaftlichen Normativität der Normenforschung und ihrer Geschichtlichkeit erfolge kaum (Inayatullah/Blaney 2012; Weber 2014).

Tatsächlich haben sich in letzter Zeit konstruktivistische NormenforscherInnen jedoch zunehmend selbst-reflexiv gegenüber der eigenen Forschung gezeigt (Price 2008a). So halten im Herausgeberband von Richard Price Moral Limit and Possibility in World Politics (2008b) unter anderem Kathryn Sikkink, Martha Finnemore oder Chris Reus-Smit dazu an, die Normativität ihrer eigenen Forschung kritisch zu reflektieren. Jedoch kann Prices Fokus auf moralischen Fortschritt durch die Etablierung von Normen einer postkolonialen Kritik (Inayatullah/Blaney 2012) oder den Anforderungen normativer Theorie (Weber 2014) nicht standhalten. Die Frage nach der Umstrittenheit des moralischen Fortschritts unterstreicht den Stellenwert von Normenforschung selbst als politische Praxis (Engelkamp et al. 2013, 2016). Somit hat die Art der praktizierten Forschung nicht nur Einfluss auf den Untersuchungsgegenstand, sondern reflektiert auch eine dezidiert normative Position mit realen politischen Konsequenzen (Engelkamp et al. 2012).

\section{Neue Wege in der Normenforschung durch metatheoretische Reflexion}

Wie der vorangegangene Abschnitt zeigt, haben die Auseinandersetzungen mit frühen Arbeiten der Normenforschung insbesondere durch unterschiedliche konstruktivistische Lesarten bislang vernachlässigte Perspektiven explizit gemacht. Gleichzeitig steigt damit der Bedarf an Verständigung zwischen unterschiedlichen kritischen Lesarten. Kritische Ansätze bergen dann großes Potenzial für theoretische Weiterentwicklung, wenn sie ihre eigenen theoretischen und methodischen Herangehensweisen metatheoretisch reflektieren und somit ihre Wissensbestände kritisch hinterfragen, so dass konstruktivistische Normenforschung zu einem wirklichen Ort des Austausches verschiedener Lager werden kann. Dies ist insbesondere für die eigene Normativität kritischer Normenforschung relevant, da sie sich mit Fragen des normativen Wandels und der Herausbildung legitimer Formen von politischer Ordnung befasst. Wie im vorhergehenden Abschnitt dargestellt, sind die Bedingungen, unter denen Wissen über normativen Wandel entsteht, keine bloßen Repräsentationen einer Wirklichkeit »da draußen«. Vielmehr beeinflusst der analytische Fokus der WissenschaftlerInnen ihre Konzepte, ihre methodologische Verortung, ihre Welt- 
sicht und die Art und Weise, wie Bedeutungen über die soziale Wirklichkeit erst zum Gegenstand der Forschung gemacht werden.

Eine solche reflexive Wendung hin zu mehr Transparenz der eigenen Diskursposition, wie sie beispielsweise von Neumann und Neumann (2015) gefordert wird, würde innerhalb kritischer Normenforschungsansätze eine präzisere Beschreibung der Schnittstellen zwischen internationaler und lokaler Ebene erlauben. Praktiken des Widerstands und der Aushandlung von Normen sowie die Konstitution und Reproduktion unterschiedlicher Machtstrukturen, die normativen Wandel beeinflussen, würden in den Fokus rücken. Um diesen neuen Pfaden in der Normenforschung Raum zu geben, sollen sowohl die metatheoretischen Forschungsperspektiven als auch die normativen Implikationen einer theoretisch-pluralistischen Normenforschung im Zentrum dieses Bandes stehen (vgl. für diese Herangehensweise auch Albert/Lapid 1997; Jackson/Nexon 2013). Daher setzt der Band zwei inhaltliche Schwerpunkte, um die eingangs formulierten Fragen nach der Forschung über und zu Normen sowie der Möglichkeiten zur Verständigung aufzugreifen: zum einen betrachten wir Normenforschung aus einer metatheoretischen Perspektive, um zu einer Verständigung über wissenschaftstheoretische Grundlagen der IB-Normenforschung beizutragen. Zum anderen widmet sich der Band theoretischen Alternativen zur bestehenden konstruktivistischen Normenforschung.

Der Sammelband bringt erstmals verschiedene konstruktivistische Ansätze aus den deutschsprachigen IB zusammen, die sich kritisch damit auseinandersetzen, wie über Normen in den IB geforscht und wie Normenforschung konkret betrieben wird. Der vorliegende Band hat zum Ziel, die Diskussion zu neuen Wegen in der deutschen IB-Forschung zu bereichern. Für das Schlusskapitel nehmen wir daher die Metapher des Lagerfeuers wieder auf und benennen Wege, die zwischen den unterschiedlichen Feuern sichtbar werden. Mit Hilfe einer metatheoretischen Perspektive können wir die Kritikpunkte an der IB Normenforschung wieder aufgreifen und beschreiben, in welcher Weise unterschiedliche Konstruktivismen so zueinander sprechen, dass sie im Hinblick auf den Erkenntnisgewinn künftiger Forschung vielversprechend sind.

Neben einer Bestandsaufnahme der IB-Normenforschungen widmen sich die hier versammelten Beiträge den neueren Entwicklungen und insbesondere kritischen Ansätzen. Hier fällt auf, dass viele AutorInnen neben den bislang vorherrschenden handlungstheoretischen oder neo-institutionalistischen Theoriemodellen alternative, eher soziologisch inspirierte $\mathrm{Zu}$ gänge gewählt haben. Diese Beiträge werfen kritische Fragen nach Macht und Widerstand, Umstrittenheit und Hegemonie, Artikulation und Perfor- 
manz auf, die in bisherigen konstruktivistischen Ansätzen oftmals unzureichend thematisiert wurden. Hiermit geraten metatheoretische Aspekte wie die reflexive Frage nach der Normativität von Wissen und Wissenschaft, aber auch die Problematisierung der Rolle des Subjekts bei der Entstehung und Verhandlung von Normen in den Blick.

Die Beiträge schließen damit an aktuelle Entwicklungen der internationalen Forschung an und ermöglichen zudem eine Neubewertung von zentralen Problemen der konstruktivistischen Normenforschungen wie der Frage der Bedeutungskonstitution durch Praktiken oder der analytischen Fassung von normativem Wandel und Normenlokalisierung. So lässt sich - im Zuge der sogenannten linguistic und practice turns in den Sozialwissenschaften (Neumann 2002; Adler/Pouliot 2011) - eine stärkere Untersuchung der Rolle von Diskursen und diskursiven Praktiken in der Normenforschung beobachten. Methodisch und methodologisch zeigt sich diese kritische Wendung vor allem in einem stärkeren Einsatz von interpretativen Techniken wie der Diskursanalyse, Genealogie, Dekonstruktion sowie hermeneutischen oder ethno-methodologischen Ansätzen. Vor diesem Hintergrund bringt der Sammelband unterschiedliche Perspektiven auf kritische Normenforschung zusammen, um über die eigenen Forschungsperspektiven zu reflektieren und zu einem Austausch verschiedener Lager beizutragen.

\section{Die Beiträge dieses Sammelbandes}

Die Verständigung im vorliegenden Band formiert sich entlang der beiden oben formulierten Schwerpunkte, entsprechend gehen wir in zwei Schritten vor: Teil 1 leistet eine Verständigung über metatheoretische Grundlagen und versammelt Beiträge über Normenforschung, während sich Teil 2 mit neueren Ansätzen in der Normenforschung beschäftigt und theoretische Alternativen zum Normenkonzept präsentiert. Der erste Teil behandelt insbesondere die Frage, wie theoretische Konzepte und metatheoretische Annahmen ihren Analysegegenstand prägen und welche unterschiedlichen epistemologischen Perspektiven, ontologischen Annahmen und methodologischen Divergenzen sich in der Normenforschung verbergen.

In seinem Beitrag gibt Bastian Loges zunächst einen Überblick über metatheoretische Zugänge, um so unterschiedliche »Normenforschungen" entlang ihrer jeweiligen ontologischen und methodologischen Schwerpunktsetzungen verorten zu können. Aus einer wissenschaftstheoretischen Perspektive weist er auf das Auseinanderklaffen von ontologischen Annahmen und methodologischer Umsetzung in der Normenforschung hin. Sei- 
ne Bestandsaufnahme zeigt, dass trotz einer großen Offenheit gegenüber verschiedenen Forschungsperspektiven die Heterogenität der metatheoretischen Grundlagen einer Kanonisierung der Normenforschung im Weg steht.

Der Beitrag von Antonia Graf, Katharina Glaab und Stephan Engelkamp unternimmt den Versuch, das Verhältnis von drei unterschiedlichen Konzepten - Norm, Diskurs, Praktik - metatheoretisch zu vergleichen. Mit Hilfe des Begriffs des Vehikels argumentieren sie, dass in allen drei Ansätzen Bedeutungen auf unterschiedliche Art und Weise fixiert werden. Dies hat erhebliche Konsequenzen für Versuche, Dialoge zwischen den Lagerfeuern unterschiedlicher Ansätze fruchtbar zu machen.

Daniel Jacobi und Friederike Kuntz hinterfragen in ihrem Beitrag die »dualistischen Beschreibungen des Sozialen « und unterbreiten den Vorschlag, die Norm unabhängig von klassischen Intentionalitätsverständnissen zu konzipieren. Normen werden dann als ein historisch-dynamisches Produkt der Verwendung von Sprache begriffen, die als ein sowohl singulärer als auch kumulativer Effekt der Ausübung von Macht erscheinen. Normen können aus einer solchen Perspektive nicht länger als ein geteilter Standard der Angemessenheit verstanden werden, sondern müssen vielmehr als kontingente, historisch bestimmte Matrix der (Re-)Produktionsmöglichkeiten von sinnhafter Wirklichkeit konzeptionell reformuliert werden. Auf diese Weise entwickeln sie mit ihrem performativen Begriff des Sozialen eine alternative Beobachtungsperspektive, in der Normen als »ein historisch-dynamischer Effekt der Ausübung von Macht« erscheinen.

Hier knüpfen Eva Herschinger und Frank Sauer an, die sich in ihrem Beitrag mit dem Spiel von Agency und Struktur beschäftigen, das gerade in der konstruktivistischen Normenforschung von zahlreichen AutorInnen betrieben worden ist. Aufbauend auf einer Kritik dieser Forschung schlagen die AutorInnen eine $\gg$ Kombination aus Elementen pragmatistisch inspirierter Handlungstheorie und poststrukturalistisch informierter Diskurstheorie" vor, um über die Konzepte Dislokation und Krise das Wechselspiel von AkteurIn und Struktur genauer in den Blick nehmen zu können. Sie verweisen darauf, dass Struktur und Agency niemals als abgeschlossen zu betrachten sind; vielmehr konstituieren sich beide wechselseitig durch die Verbindungen, die zwischen ihnen in ihrer jeweiligen, temporär stabilen, stets prozesshaften Gestalt entstehen.

Henrik Schillinger und Holger Niemann treten in ihrem Beitrag in einen kritischen Dialog mit Antje Wieners Vorschlag eines konsistent-konstruktivistischen Normenkonzepts, der den Fokus auf die Praktiken der Bedeutungsgenerierung und die Umstrittenheit von Normen legt. Dabei verweist ihre Untersuchung "mit Wiener über Wiener hinaus" sowohl auf 
die Stärken als auch auf Herausforderungen von meaning-in-use. In diesem Sinne verstehen die Autoren Wieners Unsichtbare Verfassung der Politik als einen geeigneten Ausgangspunkt für das Projekt einer kritischen Normenforschung und verbinden ihre Kritik einer impliziten »Politik der Realität« mit dem Ziel, Wege zu deren Weiterentwicklung hin zu einem dynamischen, intersubjektiven und politischen Verständnis von Normen aufzuzeigen.

Die Beiträge im zweiten Teil des Bandes stellen neuere Ansätze innerhalb der Normenforschung vor und zeigen Alternativen auf, die sich häufig - aber nicht nur - an sprachlich-diskursiven und praxeologisch inspirierten Zugängen orientieren. So entwickelt Lisbeth Zimmermann die Fragestellung, inwiefern es vor dem Hintergrund der lokalen Adaption von Normen wie Demokratie und Rechtstaatlichkeit darum gehe, diese möglichst vollständig zu verbreiten oder ob nicht auch Spielräume für den Streit um und für die Aneignung von globalen Normen geschaffen werden sollten. Wenn dies der Fall sei, ergäbe sich daraus die Frage, wie weit eine lokale Aneignung von Normen gehen könne und wie diese zu bewerten sei. Mit Bezug auf demokratietheoretische Arbeiten von Seyla Benhabib und James Tully argumentiert Zimmermann für eine Perspektive, die den Blick auf den prozeduralen Aspekt lokaler Aneignungsprozesse richtet, da diese die demokratische Legitimität von Aneignungen durch die beteiligten AkteurInnen erhöhen könne.

Linda Wallbott behandelt in ihrer soziologisch-institutionalistischen Analyse die Verhandlungsfrage globaler Normen. Ihr Beitrag verbindet einen konzeptionellen Fokus auf diskursive Aushandlung mit einer "prozessualen und relationalen raumsoziologischen Perspektive« zum Management institutioneller Interdependenz. Sie argumentiert, dass die intentionale Konstruktion von Räumen institutioneller Wechselwirkung durch AkteurInnen nicht nur die Ausgestaltung internationaler Institutionen beeinflusst, sondern auch dominante Ordnungsvorstellungen herausfordert und Normenwandel initiiert. Die Neugestaltung von Räumlichkeit und die Verschiebung institutioneller wie normativer Grenzen in sozialen Praktiken ist dabei ein Ausdruck der kontingenten und unabgeschlossenen Natur von Normen.

Sassan Gholiagha, Hannes Hansen-Magnusson und Maren Hofius fragen in ihrem Beitrag nach dem Verhältnis von Normalität und Normativität. Dabei gehen sie von einer Perspektive aus, die die Umstrittenheit von Normbedeutungen in den Mittelpunkt des Interesses stellt. Anhand zweier Fallstudien zur Folterverbotsnorm illustrieren die AutorInnen den erkenntnistheoretischen Mehrwert des meaning-in-use Konzepts für die Normenforschung: ihr Beitrag zeigt, wie sich Bedeutungszuschreibungen 
durch soziale Praktiken vor dem Hintergrund von scheinbar eindeutig kodifizierten (Rechts-)Normen wandeln und sich Normenwandel durch eine Perspektive auf Mikrophänomene erschließt.

Judith Renner und Kai Koddenbrock schlagen jeweils alternative theoretische Herangehensweisen zum Normenkonzept vor, die eine Verständigung zwischen jüngeren heterogenen Konzeptionen, Begrifflichkeiten und Methoden ermöglichen kann. Judith Renner wählt in diesem Sinne einen poststrukturalistischen Ansatz zur Untersuchung der globalen Versöhnungsnorm. Normen sind in ihrem Beitrag Ergebnis hegemonialer diskursiver Formationen, deren Wirklichkeitspolitik es in einem ersten Schritt zu dekonstruieren gilt, um danach alternative diskursive Artikulations- und Handlungsmöglichkeiten zu rekonstruieren, wodurch eine größere Handlungsmöglichkeit der durch die Norm adressierten Subjekte erreicht werden könne.

Kai Koddenbrock widmet sich in seinem Beitrag ebenfalls der Dekonstruktion einer normalisierten diskursiven Formation: dem Staat. Am Beispiel von semistrukturierten Interviews mit Interventionspersonal rekonstruiert der Beitrag den Staat als performativen Effekt sozialer Praktiken, aber auch als wirkmächtige Norm. Koddenbrock geht dabei in seiner Analyse über die Feststellung der Wirkmächtigkeit der Staatsnorm hinaus, indem er alternative Erzählungen über den Staat im Kongo aufzeigt, die quer zu westlichen Souveränitätsnarrativen und ihren Interventionslogiken liegen.

Im abschließenden Kapitel fassen Katharina Glaab, Antonia Graf und Stephan Engelkamp die Ergebnisse der vorhergehenden Beiträge zusammen und stellen sie in einen weiteren forschungsprogrammatischen $\mathrm{Zu}$ sammenhang. Die HerausgeberInnen gehen in dem Kapitel darauf ein, inwiefern die Beiträge in metatheoretischer Hinsicht so zueinander sprechen, dass Verständigung möglich wird. Gemeinsam eruieren sie, inwiefern diese Verständigungen als neue Wege in der Normenforschung verstanden werden können und geben einen Ausblick darauf, welche Fragen angesichts politischer und gesellschaftlicher Herausforderungen neu oder anders gestellt werden können. 


\section{Literatur}

Acharya, Amitav 2004: How Ideas Spread: Whose Norms Matter? Norm Localization and Institutional Change in Asian Regionalism, in: International Organization 58: 2, 239-275.

Acharya, Amitav 2011a: Norm Subsidiarity and Regional Orders: Sovereignty, Regionalism, and Rule-Making in the Third World, in: International Studies Quarterly 55: 1, 95-123.

Acharya, Amitav 2011b: Dialogue and Discovery. In Search of International Relations Theories Beyond the West, in: Millennium - Journal of International Studies 39: 3, 619-637.

Ackerly, Brooke/True, Jacqui 2008a: Critical Perspectives on Gender and Politics. An Intersectional Analysis of International Relations: Recasting the Discipline, in: Politics \& Gender 4: 1, 156-170

Ackerly, Brooke/True, Jacqui 2008b: Reflexivity in Practice. Power and Ethics in Feminist Research on International Relations, in: International Studies Review 10: 4, 693-707.

Adler, Emanuel 1997: Seizing the Middle Ground. Constructivism in World Politics, in: European Journal of International Relations 3: 3, 319-363.

Adler, Emanuel/Pouliot, Vincent (Hrsg.) 2011: International Practices, Cambridge.

Agathangelou, Anna M./Ling, L. H. M. 2009: Transforming World Politics. From Empire to Multiple Worlds, London.

Albert, Mathias/Lapid, Yosef 1997: On Dialectic and IR Theory. Hazards of a Proposed Marriage, in: Millennium - Journal of International Studies 26: 2, 403415.

Anderl, Felix/Wallmeier, Philip 2018: Modi der Kritik des internationalen Regierens. Ein Plädoyer für immanente Kritik, in: Zeitschrift für Internationale Beziehungen 25: 1, 65-89.

Berenskötter, Felix 2014: Mehr Kreativität wagen, in: Zeitschrift für Internationale Beziehungen 21: 2, 133-139.

Brabandt, Heike/Locher, Birgit/Prügl, Elisabeth 2002: Normen, Gender und Politikwandel. Internationale Beziehungen aus der Geschlechterperspektive, in: Welttrends: 36, 11-26.

Campbell, David 1992: Writing Security. United States Foreign Policy and the Politics of Identity, Manchester.

Checkel, Jeffrey 1997: International Norms and Domestic Politics. Bridging the Rationalist-Constructivist Divide, in: European Journal of International Relations 3: 4, 473-495.

Checkel, Jeffrey 1998: The Constructivist Turn in International Relations Theory, in: World Politics 50: 2, 324-348.

Checkel, Jeffrey 1999: Norms, Institutions, and National Identity in Contemporary Europe, in: International Studies Quarterly 43: 1, 83-114. 
Deitelhoff, Nicole 2006: Überzeugung in der Politik. Grundzüge einer Diskurstheorie internationalen Regierens, Frankfurt am Main.

Deitelhoff, Nicole/Zimmermann, Lisbeth 2013: Aus dem Herzen der Finsternis. Kritisches Lesen und wirkliches Zuhören der konstruktivistischen Normenforschung. Eine Replik auf Stephan Engelkamp, Katharina Glaab und Judith Renner, in: Zeitschrift für Internationale Beziehungen 20: 1, 61-74.

Der Derian, James/Shapiro, Michael J. (Hrsg.) 1989: International/Intertextual Relations. Postmodern Readings of World Politics, Lexington.

Diez, Thomas 2005: Constructing the Self and Changing Others: Reconsidering 'Normative Power Europe', in: Millennium - Journal of International Studies 33: 3, 613-636.

Doty, Roxanne L. 1996: Imperial Encounters. The Politics of Representation in North-South Relations, Minneapolis.

Engelkamp, Stephan/Glaab, Katharina 2015: Writing Norms: Constructivist Norm Research and the Politics of Ambiguity, in: Alternatives: Global, Local, Political 2015: 40 (3-4), 201-218.

Engelkamp, Stephan/Glaab, Katharina/Renner, Judith 2012: In der Sprechstunde. Wie (kritische) Normenforschung ihre Stimme wiederfinden kann, in: Zeitschrift für Internationale Beziehungen 19: 2, 101-128.

Engelkamp, Stephan/Glaab, Katharina/Renner, Judith 2013: Ein Schritt vor, zwei Schritte zurück? Eine Replik auf Nicole Deitelhoff und Lisbeth Zimmermann, in: Zeitschrift für Internationale Beziehungen 20: 2, 105-118.

Engelkamp, Stephan/Glaab, Katharina/Renner, Judith 2016: Normalising Knowledge? Constructivist Norm Research as Political Practice, in: European Review of International Studies 2016: 3: 3, 52-62.

Epstein, Charlotte 2008: The Power of Words in International Relations. Birth of an Anti-Whaling Discourse, Cambridge, Massachusetts.

Epstein, Charlotte 2011: Who Speaks? Discourse, the Subject and the Study of Identity in International Politics, in: European Journal of International Relations 17: 2, 327-350.

Epstein, Charlotte 2012a: Interrogating the Use of Norms in International Relations. An Introduction, in: International Studies Perspectives 13: 2, 121-122.

Epstein, Charlotte 2012b: Stop Telling Us How to Behave. Socialization or Infantilization?, in: International Studies Perspectives 13: 2, 135-145.

Epstein, Charlotte 2014: The Postcolonial Perspective: An Introduction, in: International Theory 6: 2, 294-311.

Epstein, Charlotte/Zarakol, Ayse/Gallagher, Julia/Shilliam, Robbie/Jabri, Vivienne 2014: Forum: Interrogating the Use of Norms in International Relations. Postcolonial Perspectives, in: International Theory 6: 2, 293.

Fearon, James/Wendt, Alexander 2008: Rationalism v. Constructivism. A Skeptical View, in: Carlsnaes, Walter/Risse, Thomas/Simmons, Beth A. (Hrsg.): Handbook of International Relations, London, 52-72.

Finnemore, Martha/Sikkink, Kathryn 1998: International Norm Dynamics and Political Change, in: International Organization 52: 4, 887-917. 
Fuchs, Doris/Glaab, Katharina 2011: Material Power and Normative Conflict in Global and Local Agrifood Governance: The Lessons of 'Golden Rice' in India, in: Food Policy 36: 6, 729-735.

Gallagher, Julia 2014: Chopping the World into Bits. Africa, the World Bank, and the Good Governance Norm, in: International Theory 6: 2, 332-349.

Goldstein, Judith/Keohane, Robert O. (Hrsg.) 1993: Ideas and Foreign Policy. Beliefs, Institutions, and Political Change, Ithaca.

Graf, Antonia 2016: Diskursive Macht. Transnationale Unternehmen im Nachhaltigkeitsdiskurs, Baden-Baden.

Grovogui, Siba N. 2010: Postcolonialism, in: Dunne, Timothy/Kurki, Milja/Smith, Steve (Hrsg.): International Relations Theories. Discipline and Diversity, Oxford, 238-256.

Guzzini, Stefano 2000: A Reconstruction of Constructivism in International Relations, in: European Journal of International Relations 6: 2, 147-182.

Hansen-Magnusson, Hannes 2020: International Relations as Politics Among People: Hermeneutic Encounters and Global Governance, London and New York.

Hansen-Magnusson, Hannes/Vetterlein, Antje/Wiener, Antje 2020. The Problem of Non-Compliance: Knowledge Gaps and Moments of Contestation in Global Governance, in: Journal of International Relations and Development: 23: 3, 636-656.

Henderson, Errol A. 2015: Hidden in Plain Sight: Racism in International Relations Theory, in: Anievas, Alex/Manchanda, Nivi/Shilliam, Robbie (Hrsg.): Race and Racism in International Relations: Confronting the Global Colour Line, London and New York, 19-43. Hofferberth, Matthias/Weber, Christine 2015: Lost in Translation. A Critique of Constructivist Norm Research, in: Journal of International Relations and Development: 18: 1, 75-103.

Hofius, Maren/Wilkens, Jan/Hansen-Magnusson, Hannes/Gholiagha, Sassan 2014: Den Schleier lichten? Kritische Normenforschung, Freiheit und Gleichberechtigung im Kontext des »Arabischen Frühlings«. Eine Replik auf Engelkamp/Glaab/ Renner, Ulbert und Deitelhoff/Zimmermann, in: Zeitschrift für Internationale Beziehungen 21: 2, 85-105.

Holzinger, Katharina/Knill, Christoph/Jörgens, Helge (Hrsg.) 2007: Transfer, Diffusion und Konvergenz von Politiken (PVS-Sonderheft), Wiesbaden.

Holzscheiter, Anna. 2018: Affectedness, Empowerment and Norm Contestation Children and Young People as Social Agents in International Politics, in: Third World Thematics 3: 5-6, 645-663.

Inayatullah, Naeem/Blaney, David L. 2004: International Relations and the Problem of Difference, New York.

Inayatullah, Naeem/Blaney, David L. 2012: The Dark Heart of Kindness: The Social Construction of Deflection, in: International Studies Perspectives 13: 2, 164175.

Jabri, Vivienne 2014: Disarming Norms. Postcolonial Agency and the Constitution of the International, in: International Theory 6: 2, 372-390. 
Jackson, Patrick T./Nexon, Daniel H. 2013: International Theory in a Post-Paradigmatic Era. From Substantive Wagers to Scientific Ontologies, in: European Journal of International Relations 19: 3, 543-565.

Katzenstein, Peter J. 1996a: Introduction. Alternative Perspectives on National Security, in: Katzenstein, Peter J. (Hrsg.): The Culture of National Security. Norms and Identity in World Politics, New York, 1-32.

Katzenstein, Peter J. (Hrsg.) 1996b: The Culture of National Security. Norms and Identity in World Politics, New York.

Keck, Margaret W./Sikkink, Kathryn 1998: Activists Beyond Borders. Advocacy Networks in International Politics, Ithaca.

Keohane, Robert O. 1988: International Institutions: Two Approaches, International Studies Quarterly 32: 4, 379-396.

Klotz, Audie 1995: Norms in International Relations. The Struggle Against Apartheid, Ithaca; London.

Koddenbrock Kai 2015: Strategies of Critique in IR: From Foucault and Latour towards Marx, in: European Journal of International Relations 21: 2, 243-266.

Krasner, Stephen D. 1999: Sovereignty. Organized Hypocrisy, Princeton.

Kratochwil, Friedrich 1991: Rules, Norms, and Decisions. On the Conditions of Practical and Legal Reasoning in International Relations and Domestic Affairs, Cambridge.

Kratochwil, Friedrich 2007: Of False Promises and Good Bets: A Plea for a Pragmatic Approach to Theory Building, in: Journal of International Relations and Development 10: 1, 1-15.

Krook, Mona L./True, Jacqui 2012: Rethinking the Life Cycles of International Norms. The United Nations and the Global Promotion of Gender Equality, in: European Journal of International Relations 18: 1, 103-127.

Lapid, Yosef 1989: The Third Debate: On the Prospects of International Theory in a Post-Positivist Era, in: International Studies Quarterly 33: 3, 235-254.

MacKenzie, Megan/Sesay, Mohamed 2012: No Amnesty from/for the International. The Production and Promotion of TRCs as an International Norm in Sierra Leone, in: International Studies Perspectives 13: 2, 146-163.

March, James G./Olsen, Johan P. 1998: The Institutional Dynamics of International Political Orders. In: International Organization 52: 4, 943-969.

Milliken, Jennifer 1999: The Study of Discourse in International Relations. A Critique of Research and Methods, in: European Journal of International Relations 5: 2, 225-254.

Neumann, Cecilie B./Neumann, Iver B. 2015: Uses of the Self: Two Ways of Thinking about Scholarly Situatedness and Method, in: Millennium: Journal of International Studies 43: 3, 798-819.

Neumann, Iver B. 2002: Returning Practice to the Linguistic Turn. The Case of Diplomacy, in: Millennium: Journal of International Studies 31: 3, 627-651.

Neumann, Iver B./Sending, Ole J. 2010: Governing the Global Polity. Practice, Mentality, Rationality, Ann Arbor. 
Niemann, Holger/Schillinger, Henrik 2017: Contestation 'All the Way Down'? The Grammar of Contestation in Norm Research, in: Review of International Studies 43: 1, 29-49.

Panke, Diana/Petersohn, Ulich 2012: Why International Norms Disappear Sometimes, in: European Journal of International Relations 18: 4, 719-742.

Platenkamp, Josephus 2007: Cultural Values, Human Rights and Peacekeeping Tasks. Some Anthropological Considerations, in: Militaire Spectator 176: 3, 96104.

Platenkamp, Josephus 2013: Sovereignty in the North Moluccas. Historical Transformations, in: History and Anthropology 24: 2, 206-232.

Price, Richard M. 2008a: Moral Limit and Possibility in World Politics, in: International Organization 62: 2, 191-220.

Price, Richard M. (Hrsg.) 2008b: Moral Limit and Possibility in World Politics, Cambridge.

Renner, Judith 2013: Discourse, Normative Change, and the Quest for Reconciliation in Global Politics, Manchester.

Risse, Thomas 2003: Konstruktivismus, Rationalismus und Theorien Internationaler Beziehungen - warum empirisch nichts so heiß gegessen wird, wie es theoretisch gekocht wurde, in: Hellmann, Gunther/Wolf, Klaus Dieter/Zürn, Michael (Hrsg.): Die neuen internationalen Beziehungen. Forschungsstand und Perspektiven in Deutschland, Baden-Baden, 99-132.

Risse, Thomas 2006: Transnational Actors and World Politics, in: Carlsnaes, Walter/ Risse, Thomas/Simmons, Beth A. (Hrsg.): Handbook of International Relations, London, 255-274.

Risse, Thomas/Gränzer, Sieglinde/Jetschke, Anja/Schmitz, Hans P. 1998: Internationale Menschenrechtsnormen, transnationale Netzwerke und politischer Wandel in den Ländern des Südens, in: Zeitschrift für Internationale Beziehungen 5: 1, 541.

Risse, Thomas/Ropp, Stephen C./Sikkink, Kathryn (Hrsg.) 1999: The Power of Human Rights. International Norms and Domestic Change, Cambridge.

Risse, Thomas/Sikkink, Kathryn 1999: The Socialization of International Human Rights Norms into Domestic Practices: Introduction, in: Risse, Thomas/Ropp, Stephen C./Sikkink, Kathryn (Hrsg.): The Power of Human Rights. International Norms and Domestic Change, Cambridge, 1-38.

Rosert, Elvira/Schirmbeck, Sonja 2007: Zur Erosion internationaler Normen. Folterverbot und nukleares Tabu in der Diskussion, in: Zeitschrift für Internationale Beziehungen 14: 2, 253-287.

Sandholtz, Wayne 2008: Dynamics of International Norm Change. Rules against Wartime Plunder, in: European Journal of International Relations 14: 1, 101131.

Schimmelfennig, Frank 2001: The Community Trap. Liberal Norms, Rhetorical Action, and the Eastern Enlargement of the European Union, in: International Organization 55: 1, 47-80. 
Schimmelfennig, Frank 2005: Strategic Calculation and International Socialization. Membership Incentives, Party Constellations, and Sustained Compliance in Central and Eastern Europe, in: International Organization 59: 4, 827-860.

Shilliam, Robbie 2014: "Open the Gates Mek We Repatriate”. Caribbean Slavery, Constructivism, and Hermeneutic Tensions, in: International Theory 6: 2, 349372.

Stritzel, Holger 2014: Zwischen Internationalisierung, Nationalisierung und Europäisierung. Das zweite Jahrzehnt der ZIB (auf Deutsch), in: Zeitschrift für Internationale Beziehungen 21: 2, 141-145.

Sylvester, Christine 2000: Feminist Theory and International Relations in a Postmodern Era, Cambridge.

Sylvester, Christine 2007: Whither the International at the End of IR, in: Millennium: Journal of International Studies 35: 3, 551-573.

Sylvester, Christine 2013: Experiencing the End and Afterlives of International Relations/Theory, in: European Journal of International Relations 19: 3, 609-626.

Ulbert, Cornelia 1997: Ideen, Institutionen und Kultur. Die Konstruktion (inter-)nationaler Klimapolitik in der BRD und in den USA, in: Zeitschrift für Internationale Beziehungen 4: 1, 9-40.

Ulbert, Cornelia 2012: Vom Klang vieler Stimmen: Herausforderungen 'kritischer' Normenforschung. Eine Replik auf Stephan Engelkamp, Katharina Glaab und Judith Renner, in: Zeitschrift für Internationale Beziehungen 19: 2, 129-139.

van Kersbergen, Kees/Verbeek, Bertjan 2007: The Politics of International Norms. Subsidiarity and the Imperfect Competence Regime of the European Union, in: European Journal of International Relations 13: 2, 217-238.

Vitalis, Robert 2015: White World Order, Black Power Politics: The Birth of American International Relations, Ithaca.Waever, Ole 2010: Still a Discipline After All These Debates?, in: Dunne, Timothy/Kurki, Milja/Smith, Steve (Hrsg.): International Relations Theories. Discipline and Diversity, Oxford, 297-318.

Walker, R. B. J. 1993: Inside/Outside. International Relations as Political Theory, Cambridge.

Wallbott, Linda/Schapper, Andrea 2017: Negotiating by Own Standards? The Use and Validity of Human Rights Norms in UN Climate Negotiations, in: International Environmental Agreements: Politics, Law and Economics: 17: 2, 209-228.

Weber, Martin 2014: Between "Isses" and "Oughts". IR Constructivism, Critical Theory, and the Challenge of Political Philosophy, in: European Journal of International Relations 20: 2, 516-543.

Wendt, Alexander 1999: Social Theory of International Politics, Cambridge.

Widmaier, Wesley W./Park, Susan 2012: Differences Beyond Theory. Structural, Strategic, and Sentimental Approaches to Normative Change, in: International Studies Perspectives 13: 2, 123-134.

Wiener, Antje 2003: Die Wende zum Dialog. Konstruktivistische Brückenstationen und ihre Zukunft, in: Hellmann, Gunther/Wolf, Klaus Dieter/Zürn, Michael (Hrsg.): Die neuen internationalen Beziehungen. Forschungsstand und Perspektiven in Deutschland, Baden-Baden, 133-159. 
Wiener, Antje 2004: Contested Compliance. Interventions on the Normative Structure of World Politics, in: European Journal of International Relations 10: 2, 189-234.

Wiener, Antje 2009: Enacting Meaning-in-use. Qualitative Research on Norms and International Relations, in: Review of International Studies 35: 1, 175-193.

Wiener, Antje 2010: Zur normativen Wende in den IB. Triangulation of a Different Kind, in: Zeitschrift für Internationale Beziehungen 17: 2, 335-354.

Wiener, Antje 2014: Kontestation in verknüpften Diskursräumen, in: Zeitschrift für Internationale Beziehungen 21: 2, 147-154.

Wiener, Antje/Liste, Philip 2014: Lost Without Translation? Cross-Referencing and a New Global Community of Courts, in: Indiana Journal of Global Legal Studies 21: 1, 263-296.

Zarakol, Ayşe 2014: What Made the Modern World Hang Together. Socialisation or Stigmatisation?, in: International Theory 6: 2, 311-332.

Zehfuss, Maja 1998: Sprachlosigkeit schränkt ein. Zur Bedeutung von Sprache in konstruktivistischen Theorien, in: Zeitschrift für Internationale Beziehungen 5: 1, 109-137.

Zehfuss, Maja 2002: Constructivism in International Relations. The Politics of Reality, Cambridge.

Zimmermann, Lisbeth 2016: Same Same or Different? Norm Diffusion between Resistance, Compliance, and Localization in Post-Conflict States, in: International Studies Perspectives 17: 1, 98-115.

Zimmermann, Lisbeth 2017: Global Norms with a Local Face. Rule-of-Law Promotion and Norm Translation. Cambridge.

Zürn, Michael/Binder, Martin/Ecker-Ebrbardt, Matthias/Radtke, Katrin 2007: Politische Ordnungsbildung wider Willen, in: Zeitschrift für Internationale Beziehungen 14: 1, 129-164.

Zürn, Michael/Checkel, Jeffrey 2005: Getting Socialized to Build Bridges. Constructivism and Rationalism, Europe and the Nation-State, in: International Organization 59: 4, 1045-1079.

Zwingel, Susanne 2011: How Do Norms Travel? Theorizing International Women's Rights in Transnational Perspective, in: International Studies Quarterly 56: 1, $115-129$.

Zwingel, Susanne 2016: Translating International Women's Rights. The CEDAW Convention in Context. London. 